Original Article

\title{
The effect of bilateral trainings on upper extremities muscle activation on level of motor function in stroke patients
}

\author{
Kyung Min Lim, MA, OT ${ }^{1)}$, Jinhwa Jung, PhD, OT ${ }^{2 *}$, Sunhwa Shim, MSc, OT ${ }^{3)^{*}}$ \\ 1) Department of Occupational Therapy, Yonsei University, Republic of Korea \\ 2) Department of Occupational Therapy, Semyung University: 65 Semyung-ro, Jecheon-si, Chungbuk \\ 390-711, Republic of Korea \\ 3) Department of Occupational Therapy, College of Medical Science, Jeonju University: \\ 303 Cheonjam-ro, Wansan-gu, Jeonju-si 55069, Republic of Korea
}

\begin{abstract}
Purpose] This study was conducted in order to compare muscle activation level on the affected and unaffected limb according to the recovery level of upper limb between bilateral activity with hands clasped and bilateral activity with pilates ring. [Subjects and Methods] Twenty inpatient who have had a stroke were recruited. Subjects were divided into two groups by the Fugl-Meyer Assessment of Motor Function score of moderately recovered group and well recovered group. The muscles activation of upper extremity and Co-Contraction Ratio (CCR) were analyzed. [Results] In the muscles activation of the well group, trapezius, anterior deltoid, and triceps muscles of affected side and biceps muscles of both sides were significantly higher when activity with pilates ring than activity with hands clasped. CCR of both side in the well group was significantly decreased during activity with pilates ring and in the moderate group, CCR of affected side was significantly decreased during activity with pilates ring. [Conclusion] Bilateral activity with a pilates ring is more effective than activity with hands clasped for the facilitation of muscle activation and coordination in stroke patients.

Key words: Muscle activation, Co-contraction ratio, Stroke
\end{abstract}

(This article was submitted Jul. 16, 2016, and was accepted Aug. 23, 2016)

\section{INTRODUCTION}

Functional limitation imposed due to a paretic upper limb affects more than $80 \%$ of stroke survivors ${ }^{1)}$. Upper limb impairment is the leading cause of limitation of motor function. Therefore, restoration of upper limb function is an essential aspect of stroke rehabilitation for regaining functional independency ${ }^{2}$.

An abnormal pattern of upper limb movement may occur caused by the compensation for muscle paralysis and imbalance. By training to perform a functional task, movement re-education is used to treat the abnormal pattern of muscle weakness $^{3}$. Although rehabilitation specialists are trying various approaches to facilitate the restoration of upper limb function, rehabilitation of upper limb function remains a challenge. Consequently, a number of researchers and therapists are seeking more effective therapeutic techniques of upper limb rehabilitation to restore voluntary motor control. Bilateral training (BT) is a therapeutic technique of upper limb rehabilitation. A recent meta-analysis revealed that BT has a positive effect on poststroke upper limb rehabilitation ${ }^{4}$. BT induces motor synergy between limbs to activate the motor capacity of the affected limb. In other words, voluntary movements of the unaffected limb facilitate voluntary movements of the affected limb ${ }^{5}$. Activation of the primary and supplementary motor cortex for the unaffected limb increases voluntary muscle contraction of

*Two authors have contributed equally to this work as corresponding authors. Jinhwa Jung (E-mail: otsalt@nate.com) Sunhwa Shim (E-mail: 2000choeun@daum.net)

(C)2016 The Society of Physical Therapy Science. Published by IPEC Inc.

This is an open-access article distributed under the terms of the Creative Commons Attribution Non-Commercial No Derivatives (by-nc-nd) License $<$ http://creativecommons.org/licenses/by-nc-nd/4.0/>. 
the affected limb during symmetrical movements ${ }^{6}$.

Even though BT is performed by using both the unaffected and the affected limbs simultaneously, most studies have reported the effect of BT on the affected limb. Morris \& Wijck reported one randomized controlled trial that investigated the effect of BT on the unaffected limb. In that report, subjects were classified into two groups, the bilateral group and unilateral group divided who scored $\leq 6$ on the motor assessment ${ }^{7}$. However, no study has addressed the effect of upper limb muscle activation on the unaffected limb during bilateral activity and the comparison of change in activity between the affected and unaffected limbs.

The effect of BT on the recovery level of the upper limb remains unclear. BT includes various activities such as targeted reaching activity using a peg, grasping and bringing a glass to the mouth, picking up and placing a towel, and manipulating and playing cards. While various activities are used, it is important to ensure that the movements involve both the upper limbs ${ }^{4}$. For assessing bilateral activity, movements involving hand clasped or grasping and lifting up an instrument such as a rod are used. However, no study has investigated the difference in amounts of upper limb muscle activation between bilateral activity with hands clasped and bilateral activity while lifting an instrument.

Therefore, the purpose of the present study was to compare the muscle activation level on the affected and unaffected limbs according to the recovery level of the upper limb between bilateral activity with hands clasped and bilateral activity with a pilates ring.

\section{SUBJECTS AND METHODS}

This study was conducted to twenty patients (12 males and 8 females) with hemiparetic stroke were recruited from a rehabilitation hospital in Gyeonggi province, Republic of Korea in 2014. Subjects who have had their first ever occurred stroke were recruited. Exclusion criteria included any significant musculoskeletal condition or other neurological condition, neglect, cognitive function lesion, vision lesion, apraxia. The general characteristics of subjects are summarized in Table 1. All of the protocols used in this study were approved by the University of Semyung. Before participation, the procedures, risks, and benefits were explained and consented to all the participants, who gave their informed consent. The participants' rights were protected according to the guidelines of the University of Semyung.

All subjects were assessed with Fugl-Meyer Assessment of Motor Function (FMA) to classify them into two groups of moderately recovered patient (moderate group) who scored 9 to 44 and well recovered patient (well group) who scored 45 and above ${ }^{8,9)}$.

Subjects underwent a clinical assessment of the stroke-affected side. Spasticity was assessed of the elbow joint using the Modified Asworth Scale (MAS) based on resistance felt during extension of the elbow joint at a medium speed while the subject was in a seated position. Recovery stage was assessed using Brunnstrom's upper extremity recovery stages.

We collected, amplified, analyzed (MyoResearch Master Edition 1.06 XP, Noraxon, Scottsdale, AZ, USA) Electromyography(EMG) data from the upper trapezius, anterior deltoid, biceps brachii, triceps brachii muscles (Noraxon TeleMyo 2400T, Noraxon, Scottsdale, AZ, USA). Disposable Ag-AgCl surface electrodes (3 M Health care, USA) were attached to the skin after it had been cleaned using alcohol. Inter-electrode distance of $2 \mathrm{~cm}$, and surface electrodes were attached parallel to the muscle fiber of the subject's both side, as follows: (1) upper trapezius: slightly lateral to and halfway between the cervical spine at C-7 and the acromion; (2) anterior deltoid: anterior aspect of the arm, approximately $4 \mathrm{~cm}$ below the clavicle; (3) biceps brachii: half way along the line between the anterior crista of the acromion and the elbow joint ; and (4) triceps brachii (lateral head): halfway along the line between the posterior crista of the acromion and the olecranon at two finger widths lateral to the line ${ }^{10}$. All EMG signal were amplified, digitized at a sampling rate of $1,000 \mathrm{~Hz}$, and band-pass $(20 \mathrm{~Hz}$ to $450 \mathrm{~Hz})$ filtered. Normalization to maximal voluntary isometric contractions (MVIC) and the root mean square

Table 1. General characteristics of the subjects

\begin{tabular}{lcc}
\hline & Well group & Moderate group \\
\hline Gender (male/female) & $6 / 4$ & $6 / 4$ \\
Age (year) & $58.2 \pm 13.5$ & $58.7 \pm 9.9$ \\
Lesion side (right/left) & $7 / 3$ & $6 / 4$ \\
Duration (month) & $16.3 \pm 27.2$ & $22.1 \pm 12.9$ \\
MMSE (score) & $27.9 \pm 2.2$ & $26.3 \pm 1.7$ \\
FMA-U (score) & $59.9 \pm 6.5$ & $25.5 \pm 13.5$ \\
Recovery stage & $5.5 \pm 0.8$ & $2.5 \pm 0.5$ \\
MAS-elbow (stage) & $0.2 \pm 0.5$ & $1.4 \pm 0.4$ \\
\hline
\end{tabular}

All variables are mean \pm standard division (SD). Well group: FMA score $\geq 45$, Moderate group: FMA score 9-45, MMSE: mini mental state examination test, FMA-U: Fugl-Meyer assessment of motor function-Upper extremity scale, Recovery stage: Brunnstrom's hand function recovery stage, MAS: Modified Ashworth Scale 
(RMS) calculation were performed using MyoResearch 1.06 software (Noraxon). The level of muscle activation during exercise was expressed as \%MVIC, and indicates how much overall muscle capacity is being used ${ }^{11)}$. The co-contraction ratio (CCR) of two muscle defined as the ratio of RMS of the antagonist muscle (biceps brachii) to the RMS of the agonist muscle (triceps brachii) during the bilateral activity. CCR represent the ratio of normalized agonistic muscle activity to normalized antagonistic muscle activity during BT. A decreasing CCR value signifies a decrease in antagonist co-activation, higher coordination, and smoother movements ${ }^{12)}$.

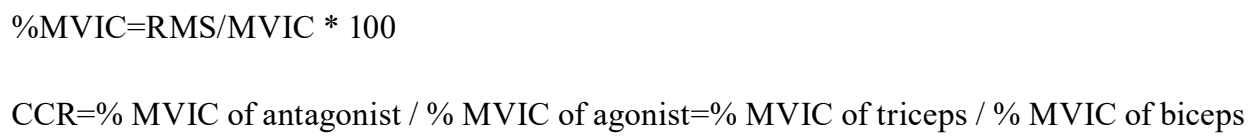

Subjects were seated in a straight-backed chair. (EMG) was performed during maximal voluntary isometric contraction of each muscle (upper trapezius, anterior deltoid, biceps and triceps brachii) using a standard manual muscle testing technique ${ }^{13}$ ) to normalize EMG amplitude according to individual differences. MVIC trials consisted of three 5-second exertion periods with a 1-minute rest period between each. The MVIC value was determined by calculating the mean peak amplitude of each MVIC trial ${ }^{11}$. After 5 minutes of rest, both hands were placed at the start point on the knee. Subjects performed two types of activities. In one task, both the arms and hands were raised to eye level and then brought down to knee with hands clasped, and in the other task, both arms were raised to eye level and then brought down to knee with the Pilates ring between hands. To control sequence effects on performance, the trial order was randomly assigned for each subject. All subjects performed both the activities, and each activity was repeated 10 times. Each measurement was repeated after a 5-minute rest period for each condition.

The measured data were analyzed using SPSS software (version 12.0 for Windows). Descriptive statistics were used to analyze general characteristics of the subjects and paired samples t-test was used to test the difference of muscle activity in each training.

\section{RESULTS}

Amounts of muscle activation (\%MVIC) in each group are shown in Table 2. In the well group, the trapezius, anterior deltoid, triceps muscles of affected limb and biceps muscles of both side were significantly higher when bilateral activity with Pilates ring than bilateral activity with hands clasped $(\mathrm{p}<0.05)$. CCR in the well group was significantly decreased during bilateral activity with Pilates ring. And in the moderate group, the co-contraction ratio of affected limb was significantly decreased during bilateral activity with Pilates ring.

\section{DISCUSSION}

This study aimed to compare muscle activation level on the affected and unaffected limbs based on the recovery level of the upper limb during activity with hands clasped and activity with a pilates ring. Many studies have shown changes in the amount of muscle activity as subjects learn to perform a maximal effort task ${ }^{11,14)}$, but it described that the duration of a muscle activity is reduced ${ }^{14)}$. Kinematic improvement related to muscle activation pattern can help to clarify the relationship

Table 2. Changes in muscle activation and co-contraction ratio (CCR)

\begin{tabular}{llcccc}
\hline \multirow{2}{*}{ Item } & \multirow{2}{*}{ Subtest } & \multicolumn{2}{c}{ Well group } & \multicolumn{2}{c}{ Moderate group } \\
\cline { 3 - 5 } & & Hands clasped & Pilates ring & Hands clasped & Pilates ring \\
\hline \multirow{2}{*}{ \%MVIC (\%) } & AD & $29.4 \pm 14.3$ & $37.5 \pm 14.4^{* *}$ & $20.9 \pm 6.2$ & $27.1 \pm 9.9^{*}$ \\
affected & BB & $24.3 \pm 14.4$ & $37.4 \pm 11.6^{* *}$ & $17.9 \pm 7.3$ & $27.5 \pm 13.7^{* *}$ \\
& TB & $13.6 \pm 13.8$ & $25.5 \pm 9.7^{* *}$ & $8.0 \pm 6.1$ & $17.6 \pm 17.2$ \\
& Tr & $17.3 \pm 13.4$ & $21.9 \pm 13.2^{*}$ & $22.2 \pm 19.5$ & $23.2 \pm 16.9$ \\
\%MVIC (\%) & AD & $30.1 \pm 9.8^{*}$ & $28.4 \pm 7.9$ & $21.8 \pm 10.9$ & $23.7 \pm 12.4$ \\
non-affected & BB & $25.5 \pm 7.9^{*}$ & $23.4 \pm 10.4$ & $16.7 \pm 13.6$ & $19.6 \pm 11.9$ \\
& TB & $12.4 \pm 8.9$ & $20.3 \pm 11.0^{*}$ & $6.0 \pm 5.0$ & $11.5 \pm 8.9$ \\
CCR & Affected & $32.4 \pm 29.0^{* *}$ & $23.4 \pm 25.2$ & $24.3 \pm 19.0^{*}$ & $16.6 \pm 13.1$ \\
& Non affected & $2.85 \pm 1.89$ & $1.48 \pm 1.77^{*}$ & $5.22 \pm 3.86$ & $1.89 \pm 1.28^{*}$ \\
\hline
\end{tabular}

All variables are mean \pm standard division (SD). \%MVIC: maximal voluntary isometric, CCR: co-contraction ratio, affected: affected side, Non-affected: nonaffected side, Well group: FMA score $\geq 45$, Moderate group: FMA score 9-45, Tr: trapezius, AD: anterior deltoid, BB: biceps brachii, TB: triceps brachii, ${ }^{*} \mathrm{p}<0.05,{ }^{* *} \mathrm{p}<0.01$ 
between muscle activation and co-contraction ratio. Therefore, we confirmed the distinctions between the pattern of muscle activation in bilateral activity with hands clasped and bilateral activity with a Pilates ring based on the measured muscle activation of the biceps and triceps related to the shoulder movements.

When comparing the groups, the well group showed a significant increase in muscle activation (trapezius, anterior deltoid, triceps) of the affected limb during bilateral activity with a pilates ring compared to bilateral activity with hands clasped $(p<0.05)$. Furthermore, significant increase in muscle activation was observed (trapezius, anterior deltoid, triceps) in the unaffected limb during bilateral activity with the hands clasped compared to bilateral activity with the Pilates ring ( $\mathrm{p}<0.05)$. In the moderate group, the trapezius, anterior deltoid, and triceps on the affected limb and the triceps on the unaffected limb showed significant changes $(\mathrm{p}<0.05)$ during bilateral activity with the Pilates ring compared to bilateral activity with the hands clasped. CCR represent the ratio of normalized agonistic muscle activity to normalized antagonistic muscle activity during reaching motion. A low CCR indicates that co-activation of antagonistic muscles reduces during activity of the agonistic muscles and that muscle coordination for movement is well controlled ${ }^{12)}$. These results showed that with the exception of bilateral activity with a Pilates ring on the unaffected limb, CCR 1 or more in the well group indicates a decrease in muscle coordination during bilateral activity. In both groups, the CCR value was low during bilateral activity with the pilates ring compared with that of hands clasped. In particular, the well group showed a significant increase in bilateral activity with the Pilates ring compared with that of hands clasped in both limbs $(\mathrm{p}<0.05)$. A study by Gabriel reported a high correlation between kinematics and EMG parameters for healthy subjects ${ }^{14)}$. A decrease in kinematic variability is associated with a decrease in the coefficient of variation of CCR between agonistic and antagonistic muscles. This is similar to our results. In contrast, there was no significant difference in CCR on the unaffected limb during BT with the Pilates ring compared with bilateral activity of hands clasped in moderate group. This finding is consistent with that of Massie et al., who reported a high correlation between kinematics and muscle activation pattern on the affected limb ${ }^{15}$.

Stroke patients frequently undergo difficulties in the movement of the affected limb. Massie et al. reported that sufficient muscle activation and agonist-antagonist co-contraction are required for generating continuous reaching motions ${ }^{15}$. Thus, the facilitation of targeted muscle activation for performing activities and the activities that induce agonistic-antagonistic muscle co-contraction are essential to establish therapeutic intervention ${ }^{16,17)}$. Furthermore, bilateral activity with a Pilates ring is more effective than activity with hands clasped for the facilitation of muscle activation and coordination in stroke patients.

The limitation of this study is that the results included the changes in EMG during bilateral activity performed 10 times, but the study was not conducted as a randomized controlled trial to compare the results before and after continuous intervention. Therefore, further studies using a randomized controlled trial design are required to demonstrate the effect on bilateral activity. Furthermore, in this study, we confirmed the quantitative change in movement, but not the qualitative change. The change in musculoskeletal qualitative movement should be investigated via three-dimensional motion analysis.

\section{ACKNOWLEDGEMENT}

This paper was supported by a Semyung University Research Grant of 2015.

\section{REFERENCES}

1) Langhorne P, Coupar F, Pollock A: Motor recovery after stroke: a systematic review. Lancet Neurol, 2009, 8: 741-754. [Medline] [CrossRef]

2) Whitall J, McCombe Waller S, Silver KH, et al.: Repetitive bilateral arm training with rhythmic auditory cueing improves motor function in chronic hemiparetic stroke. Stroke, 2000, 31: 2390-2395. [Medline] [CrossRef]

3) Ryerson S, Levit K: Functional movement reeducation: a contemporary model for stroke rehabilitation. Churchill Livingstone, 1996.

4) Cauraugh JH, Lodha N, Naik SK, et al.: Bilateral movement training and stroke motor recovery progress: a structured review and meta-analysis. Hum Mov Sci, 2010, 29: 853-870. [Medline] [CrossRef]

5) Stewart KC, Cauraugh JH, Summers JJ: Bilateral movement training and stroke rehabilitation: a systematic review and meta-analysis. J Neurol Sci, 2006, 244: 89-95. [Medline] [CrossRef]

6) Cauraugh JH, Summers JJ: Neural plasticity and bilateral movements: a rehabilitation approach for chronic stroke. Prog Neurobiol, 2005, 75: 309-320. [Medline] [CrossRef]

7) Morris JH, Van Wijck F: Responses of the less affected arm to bilateral upper limb task training in early rehabilitation after stroke: a randomized controlled trial. Arch Phys Med Rehabil, 2012, 93: 1129-1137. [Medline] [CrossRef]

8) de Niet M, Bussmann JB, Ribbers GM, et al.: The stroke upper-limb activity monitor: its sensitivity to measure hemiplegic upper-limb activity during daily life. Arch Phys Med Rehabil, 2007, 88: 1121-1126. [Medline] [CrossRef]

9) Gladstone DJ, Danells CJ, Black SE: The fugl-meyer assessment of motor recovery after stroke: a critical review of its measurement properties. Neurorehabil Neural Repair, 2002, 16: 232-240. [Medline] [CrossRef]

10) Yoo IG, Lee J, Jung MY, et al.: Neck and shoulder muscle activation in farm workers performing simulated orchard work with and without neck support. Work, 2011, 40: 385-391. [Medline]

11) Wagner JM, Dromerick AW, Sahrmann SA, et al.: Upper extremity muscle activation during recovery of reaching in subjects with post-stroke hemiparesis. 
Clin Neurophysiol, 2007, 118: 164-176. [Medline] [CrossRef]

12) Busse M, Wiles C, Van Deursen R: Muscle co-activation in neurological conditions. Phys Ther Rev, 2005, 10: 247-253. [CrossRef]

13) Kendall F, McCreary EK: Muscle testing and function. Baltimore: Williams and Wilkins, 1983.

14) Gabriel DA: Changes in kinematic and EMG variability while practicing a maximal performance task. J Electromyogr Kinesiol, 2002, 12: 407-412. [Medline] [CrossRef]

15) Massie CL, Malcolm MP, Greene DP, et al.: Kinematic motion analysis and muscle activation patterns of continuous reaching in survivors of stroke. J Mot Behav, 2012, 44: 213-222. [Medline] [CrossRef]

16) Musampa NK, Mathieu PA, Levin MF: Relationship between stretch reflex thresholds and voluntary arm muscle activation in patients with spasticity. Exp Brain Res, 2007, 181: 579-593. [Medline] [CrossRef]

17) Chae J, Yang G, Park BK, et al.: Muscle weakness and cocontraction in upper limb hemiparesis: relationship to motor impairment and physical disability. Neurorehabil Neural Repair, 2002, 16: 241-248. [Medline] [CrossRef] 